\title{
Prediction of intragranular strains in metallic polycrystals with a two-level homogenisation approach: Influence of dislocation microstructure on the mechanical behaviour
}

\author{
D. Gloaguen ${ }^{*}, 1$ and M. François ${ }^{2}$ \\ ${ }^{1}$ GeM, Institut de Recherche en Génie Civil et Mécanique, Université de Nantes, \\ Ecole Centrale de Nantes, CNRS UMR 6183, 37 Boulevard de l'Université, BP 406, \\ 44602 Saint-Nazaire, France \\ ${ }^{2}$ Laboratoire des Systèmes Mécaniques et d'Ingénierie Simultanée (LASMIS FRE CNRS 2719), \\ Université de Technologie de Troyes, 12 Rue Marie Curie, BP 2060, 10010 Troyes cedex, \\ France
}

\begin{abstract}
A two-level homogenisation approach is applied to the micro-mechanical modelling of the elasto-plasticity of polycrystalline materials during various strain-path changes. The model is tested by simulating the development of intragranular strains during different complex loads. Mechanical tests measurements are used as a reference in order to validate the model. The anisotropy of plastic deformation in relation to the evolution of the dislocation structure is analysed. The results demonstrate the relevance of this approach for FCC polycrystals.
\end{abstract}

\section{Introduction}

Mechanical anisotropy has been extensively studied due to its importance in industrial processes. Metal forming may often involve intense forming sequences, leading to large strains and severe strain-path changes. Optimising such technologies requires a good knowledge of the mechanical behaviour and then a good modelling of the anisotropic plastic behaviour of deformed material.

During plastic deformation, a grain does not deform as an entity but breaks up into cell blocks which are further subdivided into cells. The different parts are separated by dislocation walls [1,2]. The grain presents a strong heterogeneous substructure, source of third order internal stresses. One problem for an efficient polycrystal model, is the capture of the plastic anisotropy and the localization of the plastic strain which are linked to the formation of an intragranular dislocation microstructure made up of cells (with low dislocation density) and walls (with high dislocation density) [3-5]. The influence of dislocation microstructure is particularly marked during sequential loadings which are usually used in metal forming [6]. A change in the strain path is associated with hardening or softening effects due to the induced plastic anisotropy during the previous loading. This phenomenon has been revealed by different experimental studies [7-9]. These results show the importance to optimise the prediction of material strain hardening for metal forming applications so as to forecast the occurrence of plastic flow localization and fracture phenomena. 
The modelling of the plastic deformation of metallic polycrystal can be carried out by deductive methods based on strain mechanisms and scale transition methods like Taylor [10] or self-consistent (SC) models [11-13]. For the local behaviour (at the grain level), the assumption of uniform deformation neglecting the formation of the dislocation microstructure is generally used. These methods are generally appropriate for monotonic and proportional loadings. These approaches allow a correct prediction of the yield surface, the crystallographic texture linked to the plastic deformation, the intergranular stresses due to the initial and the induced anisotropy ... [14, 15]. Nevertheless, this kind of description fails to explain and reproduce the mechanical behaviour during changing strain paths [16]. These methods are not, or weakly, taking into account the formation, the evolution and the stability of the induced dislocation microstructures which are strongly path dependent.

Last years, several studies have been performed to develop models which include the effects of dislocation heterogeneities on the mechanical response of a metallic material. For example, Peeters et al. [17] developed a semi-phenomenological crystal plasticity model that incorporates more details of the microstructural evolution at the grain scale (evolution of dislocation densities) and used a full-constraint Taylor model to describe the behaviour of the material. Karaman et al. [18] have used a similar approach to successfully model of the deformation of Hadfield steel with a viscoplastic SC scheme. Mughrabi [19] introduced a model in which the crystal is considered as a composite consisting of hard dislocation walls of high local dislocation density which are separated by soft regions of low local dislocation density. A good correlation is observed with X-ray diffraction data [20]. The concept of non local hardening (the yield stress of the walls increases with the plastic strain of the cells) related to the two-phase cells structure was introduced, for the first time, by Muller et al. [21, 22] and developed later by Lemoine et al. [23] with a two-scale homogenisation based on a polycrystalline model. The morphology of the dislocation structure is taken into account by the authors. The different numerical results concerning complex loading paths seem to show a better description of the influence of the dislocation structure. A comparison with various experimental data should be interesting to investigate the accuracy of this kind of scheme. The aim of the present paper is to investigate the relevance of this scheme by comparing modelisation and experimental data. Recently, Bonfoh et al. [24, 25] have developed a two-level homogenisation approach for modelling the elastoplastic behaviour of polycrystal containing intracrystalline nonshearable particles. The numerical studies show clearly the influence of the intragranular heterogeneities on the prediction of yield surfaces and residual stresses during complex loads. Theses approaches seem promising but they were not, to the knowledge of the present authors, correlated with experimental results.

In the present work, the same approach as Muller et al. or Bonfoh et al. [21, 24] is adopted in order to predict the stress state of the deformed polycrystals in correlation with the microstructure. This study is devoted to complex loading paths. It aims the numerical study of the influence of intragranular stresses on the evolution of the mechanical behaviour during changing strain paths. A micro-mechanical description of a single crystal is developed through a micro-meso transition based on the Kröner's model. The grain is considered as a two-phase material (dislocation walls and cells). Dislocation densities on each slip system are considered as internal variables and a hardening matrix taking into account the different dislocation interactions is proposed. Next, a meso-macro transition using an elasto-plastic self-consistent method (EPSC) [26] is applied to deduce the macroscopic response of the aggregate. A modification concerning the selection of active slip systems is made for the present model. This algorithm is much faster and resolves the problem of ambiguous selection of slip systems. The last part of this paper relates to the prediction of mechanical behaviour of polycrystalline material during various complex paths. Different numerical results concerning macroscopic behaviour (loading curves) as well as microscopic features (evolution of dislocation density, stored energy, micro and mesoscopic mechanical behaviour) are given in this section. The accuracy of the simulations is evaluated by referring to mechanical experiments that have been previously published $[27,28]$. The simulations based on a two-level homogenisation provide a realistic description of the overall mechanical response of polycrystalline aggregates. 


\section{Model description}

2.1 Single crystal constitutive law, micro-meso transition scheme

We consider a metallic crystal with a cubic structure. We suppose that the main principal mechanism of plastic deformation is the crystallographic glide defined by slip systems. Based on the works of Mughrabi [19], the crystallite containing a heterogeneous dislocation distribution is considered as a two-phase composite, consisting of hard dislocation-rich and soft dislocation-poor regions. The phases have the same isotropic elastic behaviour described by shear modulus $\mu$ and Poisson's ratio $v$. The grain undergoes a strain $\boldsymbol{\varepsilon}$ (with a stress $\boldsymbol{\sigma}$ ) composed of an elastic part $\boldsymbol{\varepsilon}^{\mathrm{e}}$ and a plastic part $\boldsymbol{\varepsilon}^{\mathrm{p}}$. Describing by $\varepsilon^{\mathrm{pc}}$ and $\boldsymbol{\varepsilon}^{\mathrm{pw}}$ the mean plastic strain in the dislocation cells (c) and walls (w) respectively, one can write:

$$
\boldsymbol{\varepsilon}=(1-f) \boldsymbol{\varepsilon}^{\mathrm{w}}+f \boldsymbol{\varepsilon}^{\mathrm{c}} \quad \text { and } \quad \boldsymbol{\sigma}=(1-f) \boldsymbol{\sigma}^{\mathrm{w}}+f \boldsymbol{\sigma}^{\mathrm{c}},
$$

and with an isotropic elastic behaviour:

$$
\boldsymbol{\varepsilon}^{\mathrm{p}}=(1-f) \boldsymbol{\varepsilon}^{\mathrm{pw}}+f \boldsymbol{\varepsilon}^{\mathrm{pc}},
$$

where $f$ (respectively $1-f$ ) is the volume fraction of cell interiors (respectively cell walls) in a grain.

The solution of the problem of an inclusion in a matrix proposed by Eshelby [29], Kröner [30] was adapted to the case of a two-phase composite:

$$
\boldsymbol{\sigma}^{\alpha}=\boldsymbol{\sigma}+\alpha_{\mathrm{T}} \cdot \boldsymbol{c}^{\alpha} \cdot \cdot\left(\boldsymbol{I}-\boldsymbol{s}^{\mathrm{esh}}\right) \cdot \cdot\left(\boldsymbol{\varepsilon}^{\mathrm{p}}-\boldsymbol{\varepsilon}^{\mathrm{p} \alpha}\right) .
$$

$\boldsymbol{A} \cdot \boldsymbol{B}$ denotes the double scalar product $A_{i j k l} B_{k l m n}$ using the Einstein summation convention. $\boldsymbol{c}^{\alpha}$ is the elastic constant tensor for the phase $\alpha$ (the superscript ' $\alpha$ ' stands for walls ' $w$ ' and cells ' $c$ '). $s^{\text {esh }}$ is the Eshelby tensor and $\boldsymbol{I}$ is the fourth order identity tensor. $\boldsymbol{\sigma}^{\alpha}$ corresponds to the stress tensor in the phase $\alpha$ and $\boldsymbol{\sigma}$ is the stress tensor induced in the grain. The tensor $\boldsymbol{s}^{\text {sh }}$ is evaluated based on elastic properties of the matrix and the mechanical predicted behaviour will be too "stiff". One may retain the convenience of Kröner's treatment but make a useful assumption of plastic accommodation proposed by Berveiller and Zaoui [31]. An accommodation factor $\alpha_{\mathrm{T}}$ is introduced. This crude but simple and efficient approximation permits to simulate quite well the mechanical response of real metals [31, 32]. This factor has been used in the sequel with an additional assumption concerning $\alpha_{\mathrm{T}}$. This factor is supposed to be independent of the plastic flow and $\alpha_{\mathrm{T}}=1 / 10$ [33]. In the remainder of the paper and for more clarity, the factor $\alpha_{\mathrm{T}}$ is omitted.

Taking into account the relations (1) and (2), the mean stress tensors $\boldsymbol{\sigma}^{\mathrm{c}}$ and $\boldsymbol{\sigma}^{\mathrm{w}}$ within the cells and the walls can be written as:

$$
\boldsymbol{\sigma}^{\mathrm{c}}=\boldsymbol{\sigma}+(1-f) \boldsymbol{c}^{\mathfrak{c}} \cdot \cdot\left(\boldsymbol{I}-\boldsymbol{s}^{\mathrm{esh}}\right) \cdot \cdot\left(\boldsymbol{\varepsilon}^{\mathrm{pw}}-\boldsymbol{\varepsilon}^{\mathrm{pc}}\right)
$$

and

$$
\boldsymbol{\sigma}^{\mathrm{w}}=\boldsymbol{\sigma}-f \boldsymbol{c}^{\mathrm{w}} \cdot \cdot\left(\boldsymbol{I}-\boldsymbol{s}^{\mathrm{sh}}\right) \cdot \cdot\left(\boldsymbol{\varepsilon}^{\mathrm{pw}}-\boldsymbol{\varepsilon}^{\mathrm{pc}}\right) .
$$

The plastic flow of the phase $\alpha(=\mathrm{w}, \mathrm{c})$ can take place when the Schmid criterion is verified, i.e. slip occurs if the resolved shear stress $\tau_{\alpha}^{g}$ on a system $g$ is equal to the critical value $\tau_{c \alpha}^{g}$ depending on the hardening state of the slip system. This necessary condition is insufficient, and the complementary condition, which states that the increment of the resolved shear stress must be equal to the incremental rate of the critical resolved shear stress (CRSS), has to be checked simultaneously. The resolved shear stress is defined as the projection of the mesoscopic stress tensor $\boldsymbol{\sigma}$ on the considered slip system. In small strain formulation, one has:

$$
\tau_{\alpha}^{g}=\boldsymbol{R}_{\alpha}^{g} \cdot \cdot \boldsymbol{\sigma}^{\alpha}=\tau_{c \alpha}^{g} \quad \text { and } \quad \dot{\tau}_{\alpha}^{g}=\boldsymbol{R}_{\alpha}^{g} \cdot \cdot \dot{\boldsymbol{\sigma}}^{\alpha}=\dot{\tau}_{c \alpha}^{g},
$$


where $\boldsymbol{R}_{\alpha}^{g}$ is the Schmid tensor on a system $g$. If $\dot{\gamma}_{\alpha}^{g}$ denotes the slip rate on a system $g$, the Schmid criterion is thus given by:

$$
\begin{aligned}
& \tau_{\alpha}^{g}<\tau_{c \alpha}^{g} \Rightarrow \dot{\gamma}_{\alpha}^{g}=0, \\
& \tau_{\alpha}^{g}=\tau_{c \alpha}^{g} \quad \text { and } \quad \dot{\tau}_{\alpha}^{g}<\dot{\tau}_{c \alpha}^{g} \Rightarrow \dot{\gamma}_{\alpha}^{g}=0, \\
& \tau_{\alpha}^{g}=\tau_{c \alpha}^{g} \quad \text { and } \quad \dot{\tau}_{\alpha}^{g}=\dot{\tau}_{c \alpha}^{g} \Rightarrow \dot{\gamma}_{\alpha}^{g}>0 .
\end{aligned}
$$

The main problem is to determine which combination of slip systems will be actually activated at each step of the plastic deformation path. In this case, all possible combinations of potentially active systems must be scanned to find one that satisfies the two previous $(5 \mathrm{c})$ conditions simultaneously. Because this must be done for the two phases of each grain, running time considerations become the main task of the model. Moreover, this method can give several equivalent solutions for some hardening matrix [34]. Recently, Ben Zineb et al. [35] have proposed a new formulation to resolve the problem of ambiguous selection of slip systems and reduce running time of computations [36]. Their numerical results in the case of BCC single crystals present a good agreement with the 'classic' crystal plasticity based on the CRSS. This work proposes to extend this formulation in the polycrystalline model framework.

Based on the work of Ben Zineb et al., the relations (5a), (5b) and (5c) can be expressed with the following equation:

$$
\dot{\gamma}_{\alpha}^{g}=M_{\alpha}^{g} \dot{\tau}_{\alpha}^{g} .
$$

The slip rate is linked to the resolved shear stress rate through a function $M_{\alpha}^{g}$.

This formulation is based on the crystalline rate-dependent flow rule [37] given by:

$$
\dot{\gamma}^{g}=\dot{\gamma}_{0}\left(\left|\frac{\tau^{g}}{\tau_{c}^{g}}\right|\right)^{m-1}\left(\frac{\tau}{\tau_{c}^{g}}\right) .
$$

The exponent $n=1 / m$ is consistent with a macroscopic plastic strain rate sensitivity parameter. The constant $\dot{\gamma}_{0}$ is regarded as a reference shearing rate. This power law is different from the classical rateindependent Schmid law for which flow appears as soon as the resolved shear stress reaches the critical shear stress. In this case, $\tau_{c}^{g}$ is a reference shear stress and the viscoplastic flow described by the power is more continuous: all the slip systems are active as soon as the resolved shear stress is different from zero. In our approach, the plastic evolution is described by the relation (6a). This equation is inspired by the rate-dependent flow rule (6b) but it should be noticed that the temporal variable does not play any role in this approach. It is replacing by the evolution of $\tau_{c a}^{g}$. The control of active and non-active slip isdetermined with $M_{\alpha}^{g}$. This function depends on the ratio $\tau_{\alpha}^{g} / \tau_{c \alpha}^{g}$ and is able to describe the hardening behaviour during the plastic regime. The hardening parameter $M_{\alpha}^{g}$ is given by [36]:

$$
M_{\alpha}^{g}=\beta\left[\frac{1}{2}\left(1+\operatorname{th}\left(k_{0}\left(\frac{\tau_{\alpha}^{g}}{\tau_{c \alpha}^{g}}-1\right)\right)\right)\right]\left[\frac{1}{2}\left(1+\operatorname{th}\left(k_{0} \dot{\tau}_{\alpha}^{g}\right)\right)\right]\left[\frac{1}{2}\left(1+\operatorname{th}\left(k_{0} \tau_{\alpha}^{g}\right)\right)\right],
$$

where $\beta$ and $k_{0}$ are material constants. Hyperbolic tangent function has been tested and used because it permits to reproduce the mechanical and the hardening behaviour.

In the general case, the non local hardening laws can be written for a two-phases composite as follows:

$$
\dot{\tau}_{\mathrm{cc}}^{g}=\sum_{h} H_{\mathrm{cc}}^{g h} \dot{\gamma}_{\mathrm{c}}^{h}+\sum_{h} H_{\mathrm{cw}}^{g h} \dot{\gamma}_{\mathrm{w}}^{h} \quad \text { and } \quad \dot{\tau}_{\mathrm{cw}}^{g}=\sum_{h} H_{\mathrm{wc}}^{g h} \dot{\gamma}_{\mathrm{c}}^{h}+\sum_{h} H_{\mathrm{ww}}^{g h} \dot{\gamma}_{\mathrm{w}}^{h} .
$$

$H_{\mathrm{cc}}^{g h}, H_{\mathrm{cw}}^{g h}, H_{\mathrm{wc}}^{g h}$ and $H_{\mathrm{ww}}^{g h}$ are the hardening matrices describing interactions between dislocation moving on various slip systems. For an appropriate representation of the hardening behaviour, these matrices are expressed with microstructural variables such as dislocation density. With progressing strain, a disloca- 
tion cellular arrangement expands, composed of cell walls with high dislocation density which enclose cell interiors of low dislocation density. Dislocation sources, inside the material, generate mobile dislocations which interact with dislocations in the cell walls and increase dislocation density in this region. Few dislocations accumulate within cells. Annihilation of dislocations is mainly localized inside walls due to the rearrangement of dislocations associated with the plastic strain inside walls [38]. Based on these physical processes and using the work of Essman and Mughrabi [39], the rate of increase of dislocations density inside the walls on the system $g$ is:

$$
\dot{\rho}_{\mathrm{w}}^{g+}=\frac{1}{b L^{g}} \dot{\gamma}_{\mathrm{c}}^{g}
$$

$b$ is the magnitude of the Burgers vector, $L^{g}$ is the mean free path of mobile dislocations in the cell interiors associated to the deformation system $g$. Its evolution is governed by the density of point obstacles (essentially cell walls) cutting the system $g$ and creating interactions with gliding dislocations. Therefore:

$$
L^{g}=K \frac{1}{\sqrt{\sum_{h \neq g} \rho_{\mathrm{w}}^{h}}}
$$

where $K$ is a material constant. The annihilation term which takes into account the dynamic recovery during the deformation has the following expression:

$$
\dot{\rho}_{\mathrm{w}}^{g-}=\frac{-2 y_{a}}{b} \rho_{\mathrm{w}}^{g} \dot{\gamma}_{\mathrm{w}}^{g}
$$

$y_{a}$ is proportional to the annihilation distance of dislocation dipoles. The critical resolved shear stress can be related to the dislocation densities by the hardening relation [40]:

$$
\tau_{\mathrm{c} \alpha}^{g}=\tau_{\mathrm{c} 0 \alpha}^{g}+\xi \mu b \sqrt{\sum_{h} a^{g h} \rho_{\alpha}^{h}}
$$

$\tau_{\mathrm{c} 0 \alpha}^{g}$ is the initial reference shear stress on system $g$ and $\xi$ is a material parameter. According to Franciosi et al. [40], $a^{g h}$ is a hardening matrix which terms depend on the type of interactions between dislocation families $g$ and $h$. Only two terms, $a^{g g}$ and $a_{g \neq h}^{g h}$ (respectively self- and cross-hardening parameters), will be considered. Differentiating Eq. (12) with respect to time and considering Eqs. (9)-(11), the workhardening matrices $H_{\mathrm{wc}}^{g h}$ and $H_{\mathrm{ww}}^{g h}$ are:

$$
H_{\mathrm{wc}}^{g h}=\frac{\mu \xi}{2 \sqrt{\sum_{l} a^{g l} \rho_{\mathrm{w}}^{l}}} \frac{a^{g h}}{K} \sqrt{\sum_{l \neq h} \rho_{\mathrm{w}}^{l}} \quad \text { and } \quad H_{\mathrm{ww}}^{g h}=\frac{-\xi \mu y_{a}}{\sqrt{\sum_{l} a^{g l} \rho_{\mathrm{w}}^{l}}} \rho_{\mathrm{w}}^{h} a^{g h}
$$

The evolution law of dislocation density in walls is given by the addition of Eqs. (9) and (11) with $\rho(t=0)=\rho_{0}$.

Because of the low accumulation of dislocations inside cells, $H_{\mathrm{cc}}^{g h}$ and $H_{\mathrm{cw}}^{g h}$ are simply described by a constant. The non-local hardening term $H_{\mathrm{cw}}^{g h}$ is equal to 0 . The influence of wall region on the evolution of dislocations in cells can be considered as negligible. Finally:

$$
H_{\mathrm{cc}}^{g h}=\frac{\varsigma}{\tau_{\mathrm{cc}}^{g}-\tau_{\mathrm{c} 0 \mathrm{c}}^{g}} a^{g h} \text { and } \quad H_{\mathrm{cw}}^{g h}=0
$$

During the plastic regime, the variation of the cell interior volume fraction is considered as a function of the accumulated slip through the following empirical relation. For more details, see Refs. [2, 41]:

$$
f_{\mathrm{I}}=f_{\infty}+\left(f_{0}-f_{\infty}\right) \mathrm{e}^{-\frac{\gamma_{\gamma \mathrm{cc}}^{\mathrm{acc}}}{\gamma^{\mathrm{par}}}},
$$


where $f_{0}$ is the initial volume fraction, $f_{\mathrm{I}}$ is the cell volume fraction for a grain I. $f_{\infty}$ is the saturation value at large strains. $\gamma^{\text {par }}$ is a parameter which describes the decrease rate of $f_{\mathrm{I}} \cdot \gamma_{\mathrm{cl}}^{\text {acc }}$ is the accumulated slip in the grain I. This relation allows to take into account the intergranular plastic heterogeneity. The volume fraction of cells for each crystallite develops differently according to the effect of the grain orientation. In the remainder of the paper, the cell volume fraction will be denoted $f$ instead of $f_{\mathrm{I}}$.

By using (6a) and (4), the slip rate on a system $g$ for the cell and wall regions becomes

$$
\dot{\gamma}_{\mathrm{c}}^{g}=M_{\mathrm{c}}^{g} \boldsymbol{R}_{\mathrm{c}}^{g} \cdot \dot{\boldsymbol{\sigma}}^{\mathbf{c}} \quad \text { and } \quad \dot{\gamma}_{\mathrm{w}}^{g}=M_{\mathrm{w}}^{g} \boldsymbol{R}_{\mathrm{w}}^{g} \cdot \dot{\boldsymbol{\sigma}}^{\mathbf{w}}
$$

The plastic strain rate for each phase is the following:

$$
\dot{\boldsymbol{\varepsilon}}^{p \alpha}=\sum_{h} \boldsymbol{R}_{\alpha}^{h} \dot{\gamma}_{\alpha}^{h}
$$

Using the relation (3), (4) and (17), the slip rate for each phase is given by:

$$
\begin{aligned}
& \dot{\gamma}_{\mathrm{c}}^{g}=M_{\mathrm{c}}^{g} \boldsymbol{R}_{\mathrm{c}}^{g} \cdot \dot{\boldsymbol{\sigma}}+(1-f) M_{\mathrm{c}}^{g} \boldsymbol{R}_{\mathrm{c}}^{g} \cdot \boldsymbol{c}_{\mathrm{c}} \cdot\left(\boldsymbol{I}-\boldsymbol{s}^{\mathrm{esh}}\right) \cdot\left(\sum_{h} \boldsymbol{R}_{\mathrm{w}}^{h} \dot{\gamma}_{\mathrm{w}}^{h}-\sum_{h} \boldsymbol{R}_{\mathrm{c}}^{h} \dot{\gamma}_{\mathrm{c}}^{h}\right), \\
& \dot{\gamma}_{\mathrm{w}}^{g}=M_{\mathrm{w}}^{g} \boldsymbol{R}_{\mathrm{w}}^{g} \cdot \dot{\boldsymbol{\sigma}}-f M_{\mathrm{c}}^{g} \boldsymbol{R}_{\mathrm{w}}^{g} \cdot \boldsymbol{c}_{\mathrm{w}} \cdot\left(\boldsymbol{I}-\boldsymbol{s}^{\mathrm{esh}}\right) \cdot\left(\sum_{h} \boldsymbol{R}_{\mathrm{w}}^{h} \dot{\gamma}_{\mathrm{w}}^{h}-\sum_{h} \boldsymbol{R}_{\mathrm{c}}^{h} \dot{\gamma}_{\mathrm{c}}^{h}\right) .
\end{aligned}
$$

With Eqs. (18a) and (18b), one obtains after calculations the following matrix form:

$$
\begin{aligned}
\left(\begin{array}{c}
M_{\mathrm{c}}^{g} \boldsymbol{R}_{\mathrm{c}}^{g} \cdot \dot{\boldsymbol{\sigma}} \\
M_{\mathrm{w}}^{g} \boldsymbol{R}_{\mathrm{w}}^{g} \cdot \dot{\boldsymbol{\sigma}}
\end{array}\right)= & \left(\begin{array}{cc}
\delta_{g h}+(1-f) M_{\mathrm{c}}^{g} \boldsymbol{R}_{\mathrm{c}}^{g} \cdot \boldsymbol{c}_{\mathrm{c}} \cdot\left(\boldsymbol{I}-\boldsymbol{s}^{\mathrm{esh}}\right) \cdot \boldsymbol{R}_{\mathrm{c}}^{h} & -(1-f) M_{\mathrm{c}}^{g} \boldsymbol{R}_{\mathrm{c}}^{g} \cdot \boldsymbol{c}_{\mathrm{c}} \cdot\left(\boldsymbol{I}-\boldsymbol{s}^{\mathrm{esh}}\right) \cdot \boldsymbol{R}_{\mathrm{w}}^{h} \\
-f M_{\mathrm{w}}^{g} \boldsymbol{R}_{\mathrm{w}}^{g} \cdot \boldsymbol{c}_{\mathrm{w}} \cdot\left(\boldsymbol{I}-\boldsymbol{s}^{\mathrm{esh}}\right) \cdot \boldsymbol{R}_{\mathrm{c}}^{h} & \delta_{g h}+f M_{\mathrm{w}}^{g} \boldsymbol{R}_{\mathrm{w}}^{g} \cdot \boldsymbol{c}_{\mathrm{w}} \cdot\left(\boldsymbol{I}-\boldsymbol{s}^{\mathrm{esh}}\right) \cdot \boldsymbol{R}_{\mathrm{w}}^{h}
\end{array}\right) \\
& \times\left(\begin{array}{c}
\dot{\gamma}_{\mathrm{c}}^{h} \\
\dot{\gamma}_{\mathrm{w}}^{h}
\end{array}\right)
\end{aligned}
$$

where $\delta_{g h}$ is the Kronecker's delta. It should be noticed that superscripts $g$ and $h$ vary from one to N (N: number of active systems for each phase ' $c$ ' and 'w') for each term of the expression (19). For the sake of clarity, the Eq. (19) is written in synthetic form: the variation of the superscript $g$ and $h$ is omitted.

Equation (19) can be recasted in a condensed form:

$$
\left(\begin{array}{c}
M_{\mathrm{c}}^{g} \boldsymbol{R}_{\mathrm{c}}^{g} \cdot \dot{\boldsymbol{\sigma}} \\
M_{\mathrm{w}}^{g} \boldsymbol{R}_{\mathrm{w}}^{g} \cdot \dot{\boldsymbol{\sigma}}
\end{array}\right)=\left(\begin{array}{cc}
\boldsymbol{B}_{\mathrm{cc}}^{g h} & \boldsymbol{B}_{\mathrm{cw}}^{g h} \\
\boldsymbol{B}_{\mathrm{wc}}^{g h} & \boldsymbol{B}_{\mathrm{ww}}^{g h}
\end{array}\right)\left(\begin{array}{c}
\dot{\gamma}_{\mathrm{c}}^{h} \\
\dot{\gamma}_{\mathrm{w}}^{h}
\end{array}\right) \Leftrightarrow\left(\begin{array}{c}
\dot{\gamma}_{\mathrm{c}}^{g} \\
\dot{\gamma}_{\mathrm{w}}^{g}
\end{array}\right)=\left(\begin{array}{ll}
\boldsymbol{B}_{\mathrm{cc}}^{g h} & \boldsymbol{B}_{\mathrm{cw}}^{g h} \\
\boldsymbol{B}_{\mathrm{wc}}^{g h} & \boldsymbol{B}_{\mathrm{ww}}^{g h}
\end{array}\right)^{-1}\left(\begin{array}{l}
M_{\mathrm{c}}^{h} \boldsymbol{R}_{\mathrm{c}}^{h} \cdot \dot{\boldsymbol{\sigma}} \\
M_{\mathrm{w}}^{h} \boldsymbol{R}_{\mathrm{w}}^{h} \cdot \dot{\boldsymbol{\sigma}}
\end{array}\right) \Leftrightarrow\left\{\begin{array}{l}
\dot{\gamma}_{\mathrm{c}}^{g}=\boldsymbol{T}_{\mathrm{c}}^{g} \cdot \dot{\boldsymbol{\sigma}} \\
\dot{\gamma}_{\mathrm{w}}^{g}=\boldsymbol{T}_{\mathrm{w}}^{g} \cdot \dot{\boldsymbol{\sigma}}
\end{array} .\right.
$$

The last step for a complete mechanical description of the grain response is to determine the constitutive relation which links the overall stress rate and strain rate in the grain.

The elastic strain rate is linked to the stress rate in the grain through the Hooke's law:

$$
\dot{\boldsymbol{\varepsilon}}^{\mathrm{e}}=\dot{\boldsymbol{\varepsilon}}-f \dot{\varepsilon}^{\mathrm{pc}}-(1-f) \dot{\boldsymbol{\varepsilon}}^{\mathrm{pw}}=\boldsymbol{s} \cdot \dot{\boldsymbol{\sigma}}
$$

where $\boldsymbol{s}$ is the elastic compliance tensor at the grain level.

Replacing the expressions of $\dot{\boldsymbol{\varepsilon}}^{\mathrm{p} \alpha}(17)$ and $\dot{\gamma}_{\alpha}^{g}(20)$ into (21), the linear relation is obtained:

$$
\dot{\boldsymbol{\varepsilon}}=\left[\boldsymbol{s}+\sum_{g}\left(f \boldsymbol{R}_{\mathrm{c}}^{g} \cdot \cdot \boldsymbol{T}_{\mathrm{c}}^{g}+(1-f) \boldsymbol{R}_{\mathrm{w}}^{g} \cdot \cdot \boldsymbol{T}_{\mathrm{w}}^{g}\right)\right] \cdots \dot{\boldsymbol{\sigma}}=\ell^{-1} \cdot \dot{\boldsymbol{\sigma}}
$$

$\ell$ is the elastoplastic tangent stiffness tensor. This tensor depends on active systems, elastic properties, stress rate and deformation history of each phase. 


\subsection{Global behaviour, meso-macro transition scheme}

The principles for using the SC model to predict elastoplastic deformation were proposed by Kröner and Hill [30, 42]. In the present work, the model developed in Ref. [11] is used. For a more detailed description of the EPSC model, see Refs. $[26,43]$. The polycristal model treats each grain as an inclusion embedded in a homogeneous effective medium (HEM) exhibiting the same overall response of the aggregate. Each grain is considered as an inhomogeneity with elastic (c) constant, slip mechanism characteristic of a single crystal. The polycristal is represented by a weighted discrete distribution of orientations [Euler angles $\left.\left(\varphi_{1} \phi \varphi_{2}\right)\right]$. The relation between the stress rate and the strain rate can be written for the meso- and macro-scales as follows:

$$
\dot{\boldsymbol{\sigma}}=\ell \cdot \cdot \dot{\boldsymbol{\varepsilon}} \text { and } \boldsymbol{\Sigma}=\boldsymbol{L} \cdot \dot{\boldsymbol{E}}
$$

where $\boldsymbol{L}$ is the macroscopic tangent modulus for the fictional average homogeneous medium. $\ell$ corresponds to the modulus defined in section 2.1. $\Sigma$ and $\dot{\boldsymbol{E}}$ are the macroscopic stress and strain rates respectively.

The local strain and stress rates can be obtained classically through the localization $\boldsymbol{A}$ and concentration $\boldsymbol{B}$ tensors:

$$
\dot{\boldsymbol{\varepsilon}}=\left(\boldsymbol{I}+\boldsymbol{S}^{\mathrm{esh}} \cdot \cdot \boldsymbol{L}^{-1} \cdot \cdot \Delta \ell\right)^{-1} \cdot \dot{\boldsymbol{E}}=\boldsymbol{A} \cdot \dot{\boldsymbol{E}}
$$

and

$$
\dot{\boldsymbol{\sigma}}=\ell \cdot \cdot\left(\boldsymbol{I}+\boldsymbol{S}^{\mathrm{esh}} \cdot \cdot \boldsymbol{L}^{-1} \cdot \cdot \Delta \ell\right)^{-1} \cdot \cdot \boldsymbol{L}^{-1} \cdot \cdot \boldsymbol{\Sigma}=\ell \cdot \boldsymbol{A} \cdot \boldsymbol{L}^{-1} \cdot \boldsymbol{\Sigma}=\boldsymbol{B} \cdot \boldsymbol{\Sigma},
$$

where $\boldsymbol{\Delta} \ell=\ell-\boldsymbol{L}$.

The volume averages of the local stress and strain tensors must coincide with the overall strain and stress. After some algebraic calculations, these conditions give the overall elastoplastic tensor $\boldsymbol{L}$ as a weighted average of the mesoscopic tensor $\ell$ :

$$
\boldsymbol{L}=\left\langle\ell \cdot \cdot\left[\boldsymbol{I}+\boldsymbol{S}^{\mathrm{esh}} \cdot \boldsymbol{L}^{-1} \cdot \boldsymbol{\Delta} \ell\right]^{-1}\right\rangle .
$$

Equation (25) is a non linear implicit equation because $\boldsymbol{S}^{\text {esh }}$ depends on the unknown $\boldsymbol{L}$ in the framework of EPSC theory. The Eshelby tensor is calculated by an integral equation [26] that fully takes into account the plastic anisotropy. This equation is solved iteratively. Once $\boldsymbol{L}$ is known, by specifying an overall stress or strain increment, the model can give the corresponding stress or strain tensors for each orientation. Therefore, the mechanical response of the polycrystal can be described.

\section{Numerical results}

The different simulations obtained with the micro-macro scheme presented in chapter II are compared with different experimental results. The main purpose of this part is to evaluate the capacity of the model to simulate correctly the different physical aspects of the behaviour and the induced microstructure during complex load paths. The experimental results used in this work have been published recently by research groups, on the basis of mechanical tests measurements $[27,28]$.

\subsection{AA6111 aluminium alloy}

$\mathrm{Wu}$ et al. [27] have investigated the effect of predeformation for an AA6111 aluminium alloy. To study the effect of pre-straining on the evolution of material anisotropy, a very wide sheet is stretched to 5\% strain in the transverse direction (TD). Small tensile samples are cut from the central region of the prestrained wide sample. Tensile tests are then performed on theses small tensile samples in the rolling direction $(\mathrm{RD})$. Figure 1 presents the numerical uniaxial stress-strain curve along the TD for the as- 


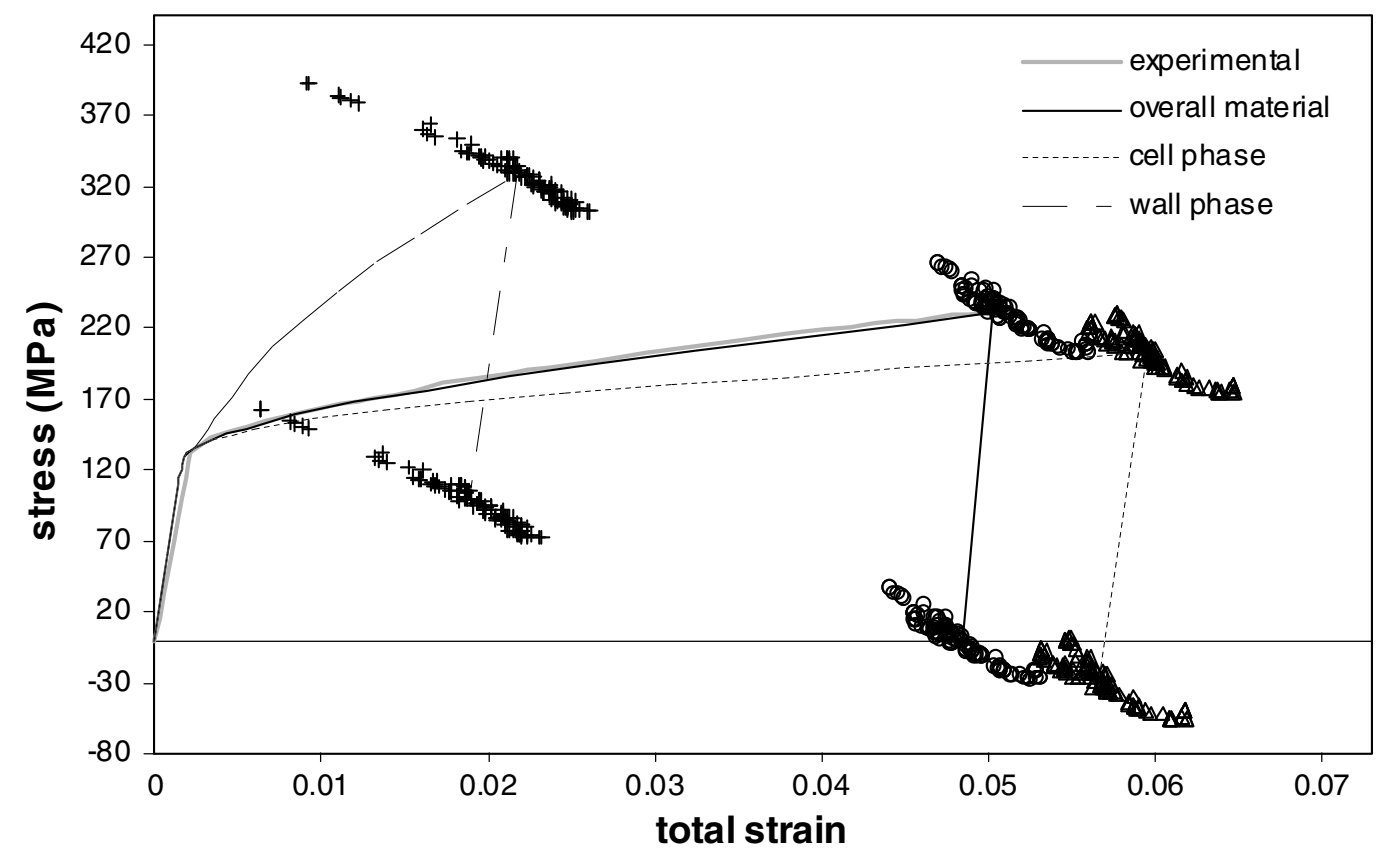

Fig. 1 Experimental curve $(-)$ and simulated pre-strain loading curve $(-)$. Scattered points represent the local strains and internal stresses heterogeneity $\left(\mathrm{O}: \sigma_{22}^{I} / \varepsilon_{22}^{I}, \Delta: \sigma_{22}^{I c} / \varepsilon_{22}^{I c},+: \sigma_{22}^{I w} / \varepsilon_{22}^{I w}\right)$ for macroscopic strains just before and after elastic unloading. Loading up to $5 \%$ total strain in the tranverse direction $x_{2}$. The experimental values are taken from Ref. [27].

received sheet with $E=5 \%$. For pre-strained sheet, Fig. 2 shows the macroscopic stress-strain curve along the $\mathrm{RD}$ with a sample deformed to $E_{11}=12 \%$. As reported by $\mathrm{Wu}$ et al., the pre-straining increases the yield stress and the work hardening is modified by residual stresses due to the first deformation and unloading.

The initial texture is taken isotropic. It is described by three randomly generated Euler's angles. Lattice rotation and thus texture changes are incorporated in the model. The model calculations were performed for a set of 100 spherical inclusions. This number of crystallites has been chosen to simulate the polycrystalline aggregate in an efficient way. For FCC metals, the plastic deformation was modelled assuming $\{110\}\langle 111\rangle$ slip systems. The same initial CRSS $\tau_{c 0}$ is taken for these deformation systems. The elastic properties are considered as isotropic. The elastic shear modulus and the Poisson's ratio are respectively equal to 62 and $0.3 \mathrm{GPa}$. The material parameters are adjusted so that the model gives a good fitting with the experimental pre-strain curve. The values of parameters associated with physical

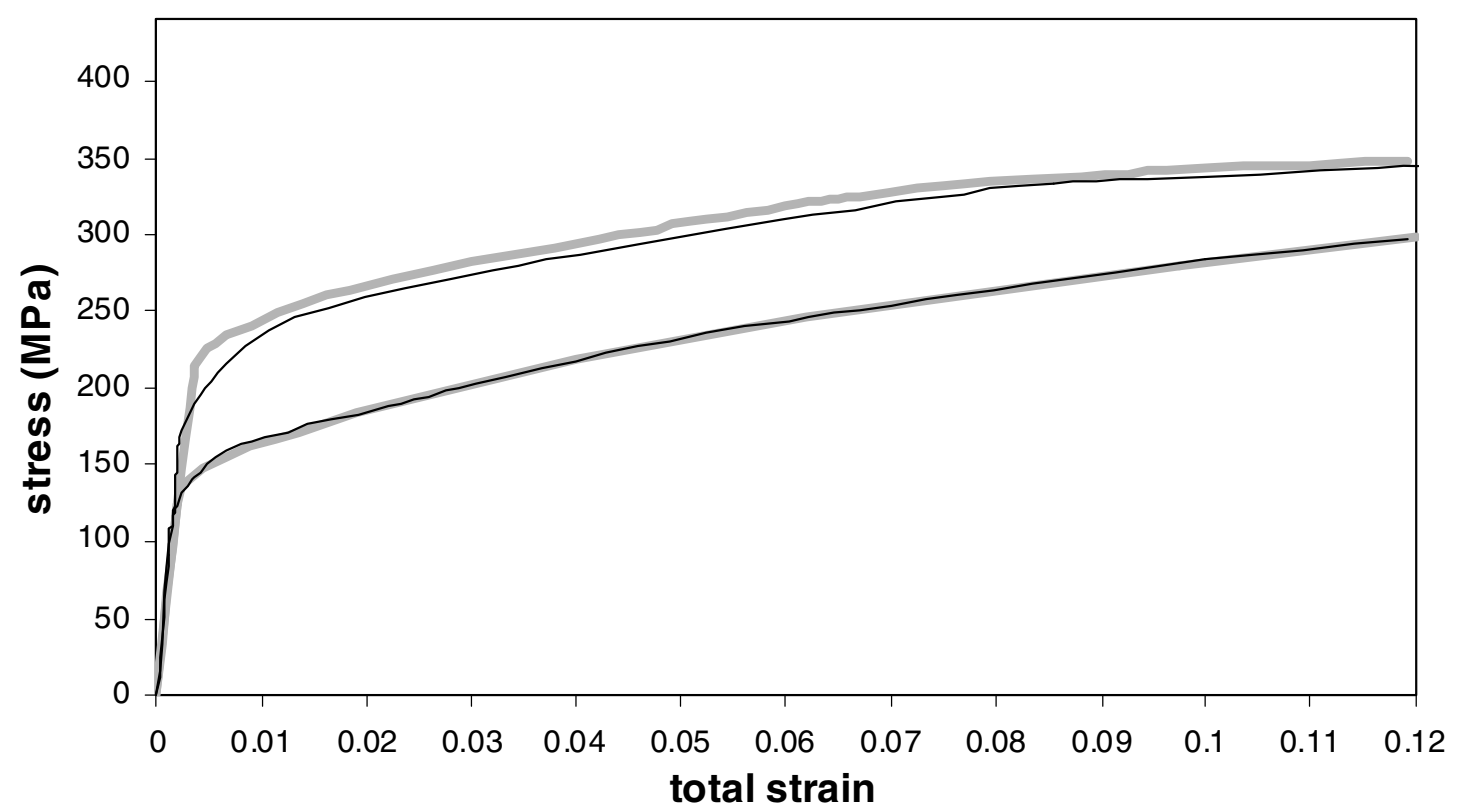

Fig. 2 Experimental ( - ) and calculated (- macroscopic stress-strain curves for the as-received sheet (a) along the TD and pre-strained sheet (b) along the RD after a 5\% preloading. 
Table 1 Material parameters used in the numerical simulations.

\begin{tabular}{lllllll}
\hline material & $\rho_{0}\left(\mathrm{~m}^{-2}\right)$ & $\xi$ & $K$ & $\tau_{c 0}(\mathrm{MPa})$ & $b(\mathrm{~m})$ & $\gamma^{\mathrm{par}}$ \\
\hline AA6111 & $10^{9}$ & 0.25 & 45 & 55 & $2.86 \times 10^{-10}$ & 3.2 \\
AISI 316L & $10^{9}$ & 0.5 & 54 & 101 & $5 \times 10^{-10}$ & 3.2 \\
\hline AA6111 & $y_{a}(\mathrm{~m})$ & $f_{0}$ & $f_{\infty}$ & $\varsigma\left(\mathrm{MPa}^{2}\right)$ & $a^{\text {gg }}$ & $a^{\text {gh }}$ \\
AISI 316L & $5.87 \times 10^{-10}$ & 0.82 & 0.75 & 1160 & 1.0 & 1.1 \\
\hline
\end{tabular}

variables $\left(\rho_{0}, y_{a} \ldots\right)$ are chosen to be coherent with those given in the literature. The parameters and constants are summarized in table 1. The stress-strain tensors, CRSS and Euler's angles obtained for the first loading after elastic unloading are used for the second tensile test. No fitting procedure is employed for this numerical simulation.

On Fig. 1, the stress-strain curve obtained with the present model and the experimental one for the first loading along the TD are compared. A good correlation is obtained with the reference data. Figure 1 also shows the internal mechanical states within cells and walls $(\alpha=\mathrm{c}, \mathrm{w})$ :

$$
\sigma_{22}^{\alpha}=\sum_{\mathrm{I}} f_{\mathrm{I}} \sigma_{22}^{I \alpha} \quad \text { and } \quad \varepsilon_{22}^{\alpha}=\sum_{\mathrm{I}} f_{\mathrm{I}} \varepsilon_{22}^{I \alpha}
$$

where $f_{\mathrm{I}}$ represents the volume fraction of grain I.

The material is initially homogeneous. This means that the elastic behaviour and the yield stress are uniform over the polycrystal. The two phases reach their yield limit at the same time which is an assumption that could be discussed. The material becomes heterogeneous since the wall phase is generated. Due to the non local hardening (through the Eq. (8)), high internal stresses are present in the wall phase. The induced microstructure appears with the plastic strain. The contrast between internal stresses within cells and walls appears coherent with the experimental one [44]. Figure 1 shows simultaneously the thirdorder stresses $\sigma_{22}^{\mathrm{I} \alpha}$ for each phase and the resulting mesoscopic stresses $\sigma_{22}^{\mathrm{I}}$ at the grain level just before and after the elastic unloading. This description permits to reveal the strong internal stresses induced in walls phase and the development of heterogeneities during plastic regime. The dislocation density in walls increases more than the one inside cells. The internal stress difference as well as the induced yield stresses are due to this phenomenon. The internal stress inside walls is approximately equal two twice the macroscopic yield stress.

The macroscopic response of the material during the second loading along the RD is presented in Fig. 2. Good agreement between experimental data and predicted results can be noted with macroscopic strain reaching $12 \%$. The macroscopic work-hardening and the slope variation of the stress-strain curve, both simulated are compatible with the reference data. The residual stresses due to the pre-straining and unloading have a significant effect on the macroscopic stress-strain behaviour through the resolved shear stress (see Eq. (4)).

Figure 3 illustrates the evolution of the dislocation densities in the walls with macroscopic strain during the first mechanical loading. This figure describes the walls dislocation density for three grains 1,2 and 3 compared to the average dislocation density $\bar{\rho}$ on the polycrystal. The grains 1 and 2 correspond, respectively, to one of the less and the most deformed crystallites. Grain 3 has an intermediate value of deformation. The initial crystallographic orientations are reported in Fig. 3. The disparity of dislocation densities from one grain to another and around $\bar{\rho}$ illustrates the intergranular plastic heterogeneity. The value of $\bar{\rho}$ reaches $10^{13} \mathrm{~m}^{-2}$ for a macroscopic strain equal to $20 \%$. This value is compatible with experimental values $[45,46]$.

These results demonstrate that the representation by a two-phase material with non-local hardening, which takes into account the creation but also the annihilation of dislocations, improves the modelling of the behaviour of material studied. 


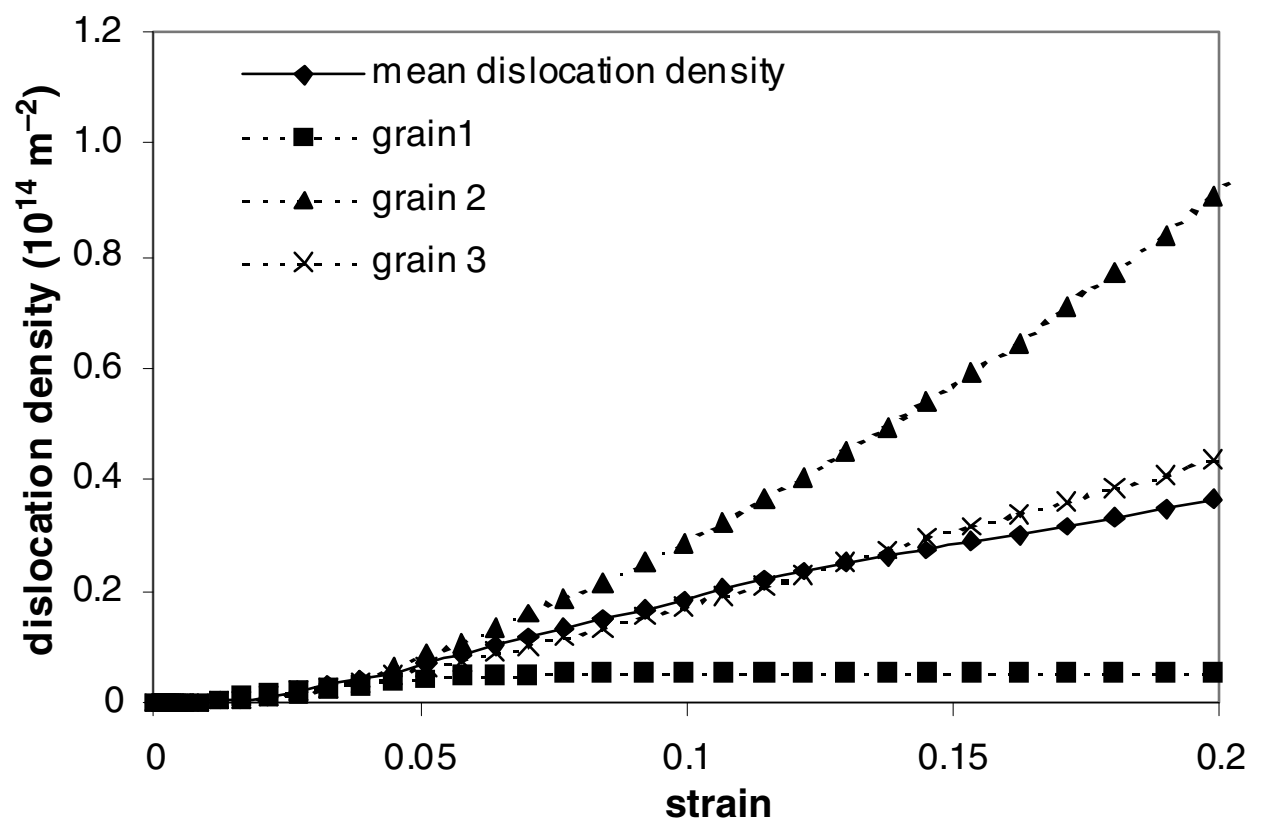

Fig. 3 Modelled total walls dislocation density in the polycrystal for the grains 1, 2 and 3 during an uniaxial loading along the TD of the aluminium sheet. Initial crystallographic orientation (Euler's angles in degree): $\{115,20,59\},\{352,97,227\},\{188,73,291\}$ for the grains 1,2 and 3 respectively.

\subsection{AISI 316L stainless steel}

In a published study [28], uni-axial Bauschinger tests in tension-compression were carried out on AISI 316L austenitic stainless steel at room temperature. Experimental strain-stress curves are shown in Fig. 4. The total pre-strain in tension is equal to $1 \%$ for the first test, 2 and $3 \%$ for the second and third ones. When the strain path is changed the flow strength and the work hardening rate will typically diverge from those expected from monotonic deformation. The decrease in initial flow stress (compared with the stress at the same equivalent strain in the monotonic loading) and the increase in work hardening rate have been explained by the internal stresses generated during pre-strain of a inhomogeneous microstructure. This phenomenon increases with the amount of the pre-strain (Fig. 4). Currently, classic

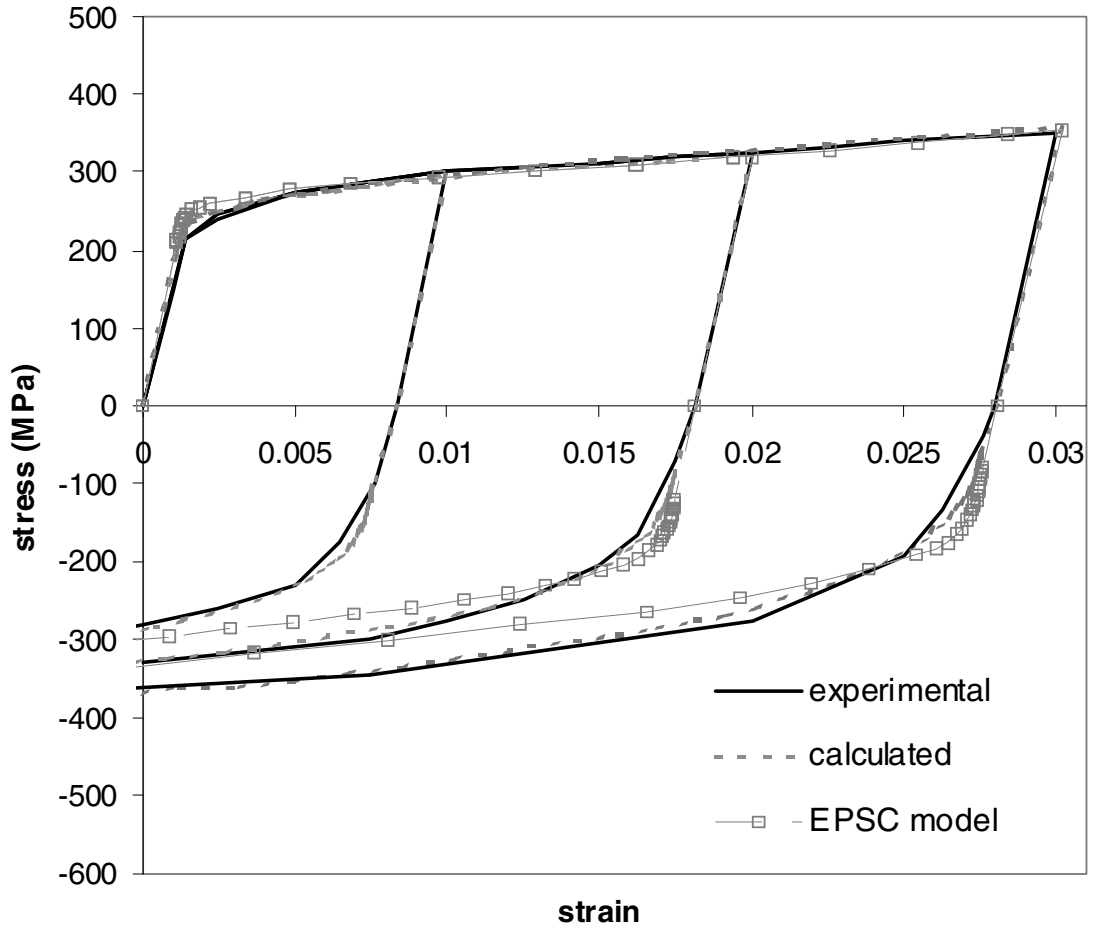

Fig. 4 (online colour at: www.pss-a.com) Comparison between experimental and simulated stress-strain curves. 
scale-transition models, like EPSC approach, fail to explain and reproduce correctly the softening effects during the compressive stage. These methods weakly take into account the formation, the evolution and the stability of induced dislocations microstructures which are strongly path dependent. The main goal of this part is to demonstrate that the present model can describe with a better accuracy the overall softening related to the dislocations microstructures in sequential paths.

The input texture consisted of a set of 100 equally-weighted lattice orientations representing a random texture. The grains were assumed to be equiaxed. Since simulations are conducted until moderate strains, no update shape of the grains were considered. No experimental data was available from the literature concerning crystallographic and morphologic textures of the tested materials. Nevertheless, it is clear that the influence of crystallographic texture is negligible compared to the effects of internal stress state introduced by the preloading. The elastic behaviour is considered isotropic and can be defined by the usual constants: $\mu=70 \mathrm{GPa}$ and $v=0.3$. The material is a FCC aggregate: the family of slip systems $\{110\}\langle 111\rangle$ permits to describe plasticity.

The material parameters $\left(a^{g h}, \tau_{\mathrm{c}} \ldots\right.$. ) have been determined by fitting the experimental tensile curve. The parameters values are listed in table 1 . The stress-strain curves with different pre-loading $(E=1,2$ and 3\%) predicted by applying the present model are plotted in Fig. 4. The numerical results are in good agreement with the experimental data obtained by Choteau et al. [28]. All experimental characteristics including the Bauschinger effect and the macroscopic work hardening are reproduced by the model for the traction and compression loading. Because of the residual stresses due to the first solicitation, the plastic regime begins in cell phase before the wall one. All the reverse loading yield stresses obtained from the model increase with pre-strain like experimental ones. As can be seen, the calculated values are lower than experimental ones but the differences are weak. The pre-strain influence on the slope of the stress-strain curve is well captured by the model.

The model is also adapted to calculate the stored energy due to the mesoscopic stresses linked to intergranular plastic heterogeneities. This energy is given by:

$$
\phi=\frac{1}{2} \sum_{\mathrm{I}} f_{\mathrm{I}} \sigma_{i j}^{\mathrm{I}} \varepsilon_{i j}^{\mathrm{Ie}}
$$

where $\varepsilon^{\text {Ie }}$ is the mesoscopic elastic strain tensor for the grain I.

The Fig. 5 shows the evolution of stored energy for two tension-compression tests with a pre-strain in tension of 2 and 10\%. During the first loading, the stored energy increases due to the development of internal stresses. After this step, an energy restitution is observed linked to the reorganisation of internal stresses. This phenomenon is more important with the pre-strain in tension. Then, the stored energy in-

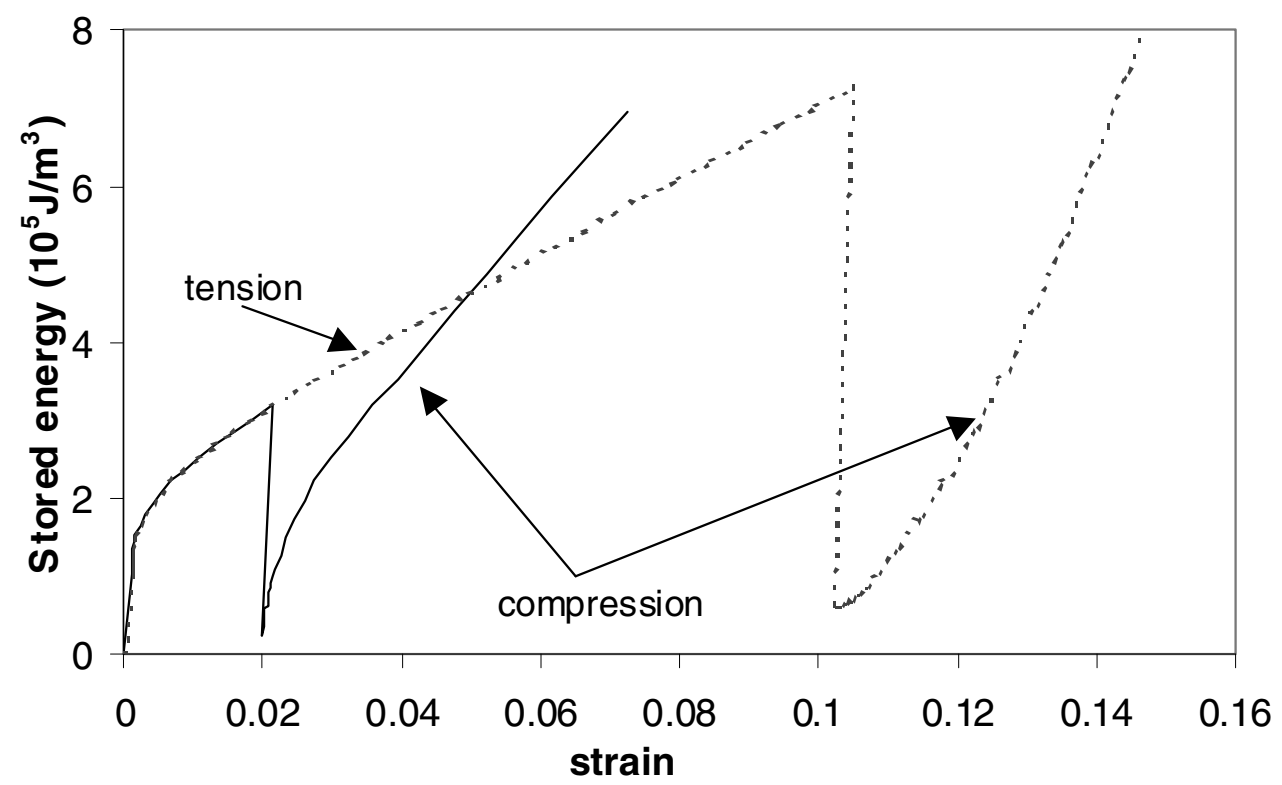

Fig. 5 (online colour at: www.pss-a.com) Evolution of the stored energy during two tensioncompression tests. (- ${ }_{-}$: pre-strain in tension up to $2 \%,(---)$ : pre-strain in tension up to $10 \%$. 
Table 2 Set of parameters of the EPSC model.

\begin{tabular}{lllll}
\hline$\mu(\mathrm{GPa})$ & $v$ & $\tau_{\mathrm{c} 0}(\mathrm{MPa})$ & $H_{1}(\mathrm{MPa})$ & $H_{2}(\mathrm{MPa})$ \\
\hline 70 & 0.3 & 108 & 375 & $3 . \mathrm{H}_{1}$ \\
\hline
\end{tabular}

creases again during the compression test. This result is in qualitative agreement with experimental and numerical data [47].

The experimental data has been also simulated using a 'classic' EPSC scheme. The set of used parameters is summarized in Table 2. The hardening matrix contains only two terms $\mathrm{H}_{1}$ and $\mathrm{H}_{2}$, corresponding to weak and strong interactions between the slip systems. In our calculations, these terms are taken constant during the loading (linear hardening). The material is described by a set of 100 spherical grains with initial random orientation. Figure 4 presents results obtained with this approach for a pre-strain of 2 and $3 \%$. The tensile curve is correctly predicted by the model. The main differences are observed for the reverse loading. The yield stress is underestimated by the model, the elasto-plastic transition and the work-hardening are not well reproduced by the simulation. The plastic activation of the grains begins for a macroscopic stress value of -130 and $-110 \mathrm{MPa}$ for a pre-strain of 2 and $3 \%$ respectively. The modified approach predicted a value of -80 and $-60 \mathrm{MPa}$ which are more coherent with experimental results.

The Fig. 6 shows the difference between the highest value of resolved shear stress and the corresponding CRSS for each grain. These values are calculated for the two models after a tensile test in tension up to $2 \%$ strain and unloaded ( $\Sigma=0 \mathrm{MPa}$ and $E=1.82 \%$ ). The Fig. 6 presents the numerical results for this phase because the cell region starts up the plastic flow during the reverse loading. Even after a moderate strain, i.e. $2 \%$, the disparity between the two approaches is marked. On average, the values predicted by the EPSC model are equal two times the ones calculated by the modified scheme.

These comparisons are important in that it shows that the difference can be explained by the internal stresses due to the first uniaxial loading. The Bauschinger effect is stronger with the nonlocal formalism because of the high third order stresses which are induced in this case compared to the second-order stresses obtained using the EPSC model. The EPSC does not reproduce quantitatively the Bauschinger effect which is strictly associated with the intragranular stresses. Due to the assumption of homogeneous plastic strain or strain rate inside the grains, the strain-induced intragranular microstructures are neglected in the EPSC model. They correspond to the heterogenization of dislocation density during the plastic flow.

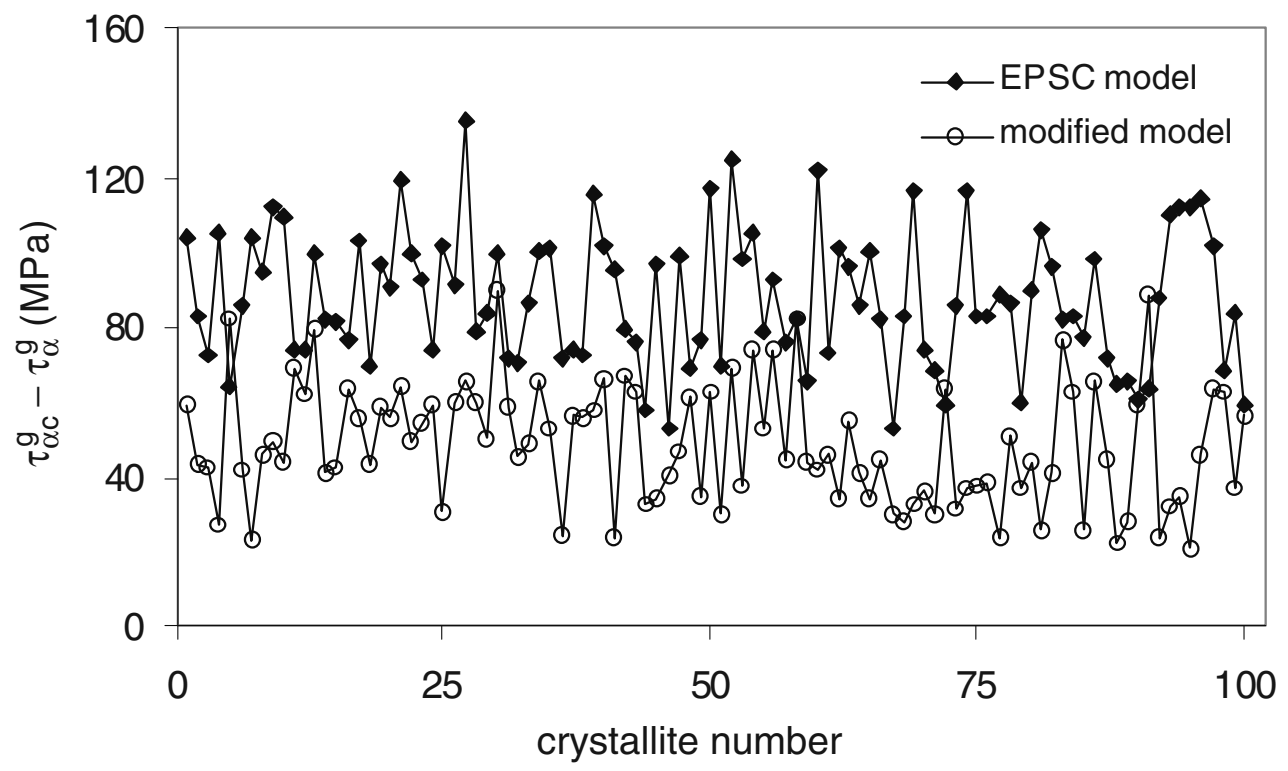

Fig. 6 Difference between the CRSS and the highest value of resolved shear stress for each grain with a macroscopic pre-strain $E=2 \%$. For the modified model, only the cell region is considered. 
The formation of dislocations substructures, i.e. cells and walls, are poorly described by the hardening matrix. This explains why the results obtained with the EPSC approach do not capture the real mechanical behaviour. The model presented in this study fits the best the experimental reverse loading. The incorporation of this physical phenomenon in the EPSC model can accurately predict the behaviour of polycrystals during complex loadings.

\section{Conclusions}

A two-level homogenisation approach has been applied to the micro-mechanical modelling of the elastoplasticity of polycrystalline materials during various strain-path changes. The model considers a cellular dislocation structure consisting of two phases: the cell walls and the cell interiors. Dislocation densities on each slip system are considered as internal variables and a non-local hardening matrix, taking into account the different dislocations interactions, is developed. The cell volume fraction is considered to decrease as a function of accumulated slip.

The predicted mechanical behaviour is compared with published experimental results and a good agreement between theory and experiment was found. Numerical results obtained at the different scales of the material show the relevance of this approach for FCC polycrystals and validate the scale transition method used. The modified self-consistent elastoplastic model is a reliable tool for the calculation of internal stresses and for derivation of single crystal properties when experiments on single crystals cannot be performed. Moreover, the model is more adapted than EPSC model to account for the intragranular stresses and their influence during a sequential loading.

\section{References}

[1] Q. Liu, D. Juul Jensen, and N. Hansen, Acta Mater. 46(16), 5819 (1998).

[2] Y. Estrin, L. S. Toth, A. Molinari, and Y. Brechet, Acta Mater. 46(15), 5509 (1998).

[3] T. Ungar, H. Mughrabi, D. Rönnpagel, M. Wilkens, Acta Metall. 32(3), 333 (1984).

[4] A. Borbély, G. Hoffmann, E. Aernoudt, and T. Ungar, Acta Mater. 45(1), 89 (1997).

[5] G. Winther and D. Juul Jensen, Comput. Mater. Sci. 9, 251 (1997).

[6] G. Boehler and A. S. Khan, Anisotropy and localization of plastic deformation (Elsevier, Amsterdam, 1991).

[7] B. K. Chun, J. T. Jinn, and J. K. Lee, Int. J. Plast. 18, 571 (2002).

[8] S. Bouvier, J. L. Alves, M. C. Oliveira, and L. F. Menezes, Comput. Mater. Sci. 32, 301 (2005).

[9] K. H. Kim and J. J. Yin, J. Mech. Phys. Solids 45, 841 (1997).

[10] T. M. Holden, R. A. Holt, and P. Clarke, J. Neutron Res. 5, 241 (1997).

[11] D. Gloaguen, M. François, R. Guillén, and J. Royer, Acta Mater. 50, 871 (2002).

[12] J. W. L. Pang, T. M. Holden, P. A. Turner, and T. E. Masson, Acta Mater. 47, 373 (1999).

[13] P. Zattarin, P. Lipinski, and A. Rosochowski, Int. J. Mech. Sci. 46, 1377 (2004).

[14] D. Gloaguen, M. François, and R. Guillén, J. Appl. Crystallogr. 37, 934 (2004).

[15] B. Clausen, T. Lorentzen, and T. Leffers, Acta Mater. 46(9), 3087 (1998).

[16] M. R. Daymond, C. N. Tome, and M. A. M. Bourke, Acta Mater. 48, 553 (2000).

[17] B. Peeters, M. Seefeldt, C. Teodosiu, S. R. Kalidindi, P. Van Houtte, and E. Aernoudt, Acta Mater. 19, 1607 (2001).

[18] I. Karaman, H. Sehitoglu, A. J. Beaudoin, Y. I. Chumlyakov, H. J. Maier, and C. N. Tome, Acta Mater. 18, $2031(2000)$.

[19] H. Mugrabi, phys. stat. sol. (a) 104, 107 (1987).

[20] T. Ungar, H. Mughrabi, D. Rönnpagel, and M. Wilkens, Acta Metall. 32(3) 333 (1984).

[21] D. Muller, X. Lemoine, and M. Berveiller, J. Eng. Mat. Technol. 116, 378 (1994).

[22] X. Lemoine, M. Berveiller, and D. Muller, Mater. Sci. Forum 157-162, 1821 (1994).

[23] F. David, I. Aubert, X. Lemoine, and M. Berveiller, Comput. Mater. Sci. 9, 188 (1997).

[24] N. Bonfoh, A. Carmasol, and P. Lipinski, Int. J. Plast. 19, 1167 (2003).

[25] N. Bonfoh, P. Lipinski, A. Carmasol, and S. Tiem, Int. J. Plast. 20, 85 (2004).

[26] P. Lipinski, and P. M. Berveiller, Int. J. Plast. 5, 149 (1989).

[27] P. D. Wu, S. R. Mac Ewen, D. J. Lloyd, M. Jain, P. Tugcu, and K. W. Neale, Int. J. Plast. 21, 723 (2005). 
[28] M. Choteau, P. Quaegebeur, and S. Degallaix, Mech. Mater. (2005), in press.

[29] J. D. Eshelby, Proc. R. Soc. A 241, 376 (1957).

[30] E. Kröner, Acta Metall. 9, 155 (1961).

[31] M. Berveiller and A. Zaoui, J. Mech. Phys. Solids 26, 325 (1979).

[32] C. Schmitt, P. Lipinski, and M. Berveiller, Int. J. Plast. 13, 183 (1997).

[33] C. Beradai, M. Berveiller, and P. Lipinski, Int. J. Plast. 3, 143 (1987).

[34] P. Zattarin, A. Baczmanski, P. Lipinski, and K. Wierzbanowski, Arch. Metall. 45, 163 (2004).

[35] T. Ben Zineb, S. Arbab Chirani, and M. Berveiller, Fifteenth French conference of mechanic, Nancy, France, 2001.

[36] D. Gloaguen, T. Berchi, E. Girard, and R. Guillen, phys. stat. sol. (a) 203, R12 (2006).

[37] U. F. Kocks, C. N. Tomé, and H. R. Wenk, Texture and anisotropy (Cambridge University Press, 1998).

[38] E. Nes, Prog. Mater. Sci. 41, 129 (1998).

[39] U. Essmann and H. Mughrabi, Philos. Mag. 40(6), 731 (1979).

[40] P. Francioisi, Acta Metall. 33(98), 1601 (1985).

[41] L. S. Toth, Comput. Mater. Sci. 32, 568 (2005).

[42] R. Hill, J. Mech. Phys. Solids 13, 89 (1965).

[43] P. A. Turner, N. Christodoulou, C. N. Tomé, Int. J. Plast. 11(3), 251 (1995).

[44] T. Ungar and I. Groma, J. Appl. Crystallogr. 22, 26 (1989).

[45] F. Roters, D. Raabe, and G. Gottstein, Acta Mater. 48(41), 81 (2000).

[46] E. Nes, K. Marthinsen, and B. Ronning, J. Mater. Process. Technol. 117, 333 (2001).

[47] J. Krier, Ph.D. thesis, University of Metz, France (1993). 